\title{
Schwinger pair production correction in thermal system
}

\author{
Y. L. Wang, ${ }^{1}$ H. B. Sang, ${ }^{1}$ and B. S. Xie $\odot^{1,2, *}$ \\ ${ }^{1}$ Key Laboratory of Beam Technology of the Ministry of Education, \\ and College of Nuclear Science and Technology, Beijing Normal University, Beijing 100875, China \\ ${ }^{2}$ Beijing Radiation Center, Beijing 100875, China
}

(Received 1 October 2019; revised manuscript received 26 November 2019; published 20 December 2019)

\begin{abstract}
In this paper, we give formal results of Schwinger pair production correction in thermal systems with external background field by using the evolution operator method of thermofield dynamics, in which especially tree-level correction of thermal photons is considered, within the approach of linear response to an effective mass shift. We consider initial systems in two types of vacuums as zero temperature and thermal vacuum, respectively, with the correction of thermal photons included or not. For an example, we give results of these corrections to pair production for a constant external background electric field.
\end{abstract}

DOI: 10.1103/PhysRevD.100.116016

\section{INTRODUCTION}

In an early work of Schwinger, it is shown that a very strong constant electric field decays to real charged pairs [1]. The produced charged pairs are observable but not observed directly yet for the absence of strong electric field near the critical field strength $E_{c}=m_{e}^{2} c^{3} / e \hbar=1.3 \times$ $10^{16} \mathrm{~V} / \mathrm{cm}$ in laboratories. Recently, a series of papers showed that direct tests of vacuum decay are planned in the near future [2-4]. Besides, some calculations indicate thermal enhancement of pair production [5-10], and it is similar to dynamically assisted Schwinger pair production [11]; consequently, an experiment on thermal assisted pair production is proposed [12].

The thermal gauge field theories have been constructed and used in quantum field-theoretical phenomena [13-17] and mainly summarized in imaginary- [18] or real-time [19] form. For thermal correction to Schwinger pair production, various methods obtain identical results at zero temperature [20] but have distinct results at finite temperature for thermal pair production studied in different mechanisms. Early discussion [21-23] combined with Schwinger's approach proved that there is no thermal contribution to the imaginary part of an effective one-loop action. But recent papers using the worldline instants technique, in which charged particles absorb thermal photons to reach maximum decay at a specific temperature

\footnotetext{
*Corresponding author. bsxie@bnu.edu.cn

Published by the American Physical Society under the terms of the Creative Commons Attribution 4.0 International license. Further distribution of this work must maintain attribution to the author(s) and the published article's title, journal citation, and DOI. Funded by SCOAP ${ }^{3}$.
}

[5-10], prove that the thermal effect enhances pair production. These also indicate that there is a temperature threshold $T_{c}=q E / 2 \pi m$ [7] and discrete resonant peaks $T_{*}=n q E / 2 \pi m[9]$ in a constant electric field $E$, where $q$ and $m$ are charge and mass. Moreover, Kim and Lee's work [24] using the operator method and Gavrilov et al.'s works $[25,26]$ using the density matrix method show that there are thermal corrections in thermal QED pair production. Here, we would like to emphasize the following three points. First, the Schwinger pair production is a nonequilibrium process, particularly when the field strength is greater than temperature. Second, in imaginary-time form, the asymptotic structure of charged particles is changed by inserting the thermal conditions in the Lagrangian, and the redefinition of asymptotic states may cause a counting problem [27]. Last, the relation of the decay rate, effective Lagrangian, and pair production number $\Gamma(A)=2 \operatorname{Im} \mathcal{L}_{\text {eff }}(A)=$ $\pm \sum_{\mathbf{k}} \ln \left(1 \pm \mathcal{N}_{\mathbf{k}}\right)$ [1] changes at finite temperature in realtime form [28]. Therefore, at finite temperature, the imaginary part of effective Lagrangian may not be equal to the measured pair production rate. Furthermore, in general thermal QED systems, the thermal photons are present and usually are considered in loop corrections but ignored in tree-level calculations. We notice that they also influence the structure of vacuum, consequently affecting the result of pair production.

Therefore, to obtain a measurable pair production rate at finite temperature, and to make a distinction among thermal charged particles, thermal photons, and external electromagnetic fields, we use the operator method of thermofield dynamics (TFD), adding an effective mass shift approach. The core of our paper is to combine Bogoliubov transformation, which depends on the solution of the KleinGordon equation or Dirac equation under external fields [24]; thermal transformation, which depends on the choice 
of inequivalent thermal vacuums; and effective mass corrections, which concern the effect of thermal photons. Our approaches adopt almost-independent approximation for particles; consequently, all interactions among charged particles are ignored, and the single-particle equations have the same accuracy with the one-loop effective Lagrangian [24], and the high-order corrections are negligible [29]. In Dirac vacuum, a negative energy (frequency) electron absorbs photons to become electrons, and its in and out states depend on background fields, which are assumed to consists of an external space-time dependent field, $A_{\mu}^{e x}(x)$, and temperature-dependent field, $A_{\mu}^{\gamma}\left(T_{\gamma}\right)$, so that $A_{\mu}=A_{\mu}^{e x}(x)+A_{\mu}^{\gamma}\left(T_{\gamma}\right)$. Assuming that thermal fields are turned off when $t \rightarrow \infty$ (before measurement) and employing semiclassical mass shift $m^{* 2}=m^{2}-q^{2}\left\langle A^{\gamma, \mu} A_{\mu}^{\gamma}\right\rangle$ to reflect the corrections of thermal photon fields, we find that $T_{\gamma}$ appears in the exponent, which decreases the threshold of tunneling so that it enhances the rate of pair production.

The paper is organized as follows. In Sec. II, we revisit the pair production in vacuum and then consider it in thermal charged particles, thermal photons, and their mixture. We will show their similar results only with a change in thermal photons and the mixture when temperature is small $T_{\gamma} \ll m$. In Sec. III, we apply the method to a constant electric field and give the integral form and lowtemperature polynomial form. In Sec. IV, we summarize our results and point out future directions. We use the natural units in which $c=\hbar=k_{B}=\varepsilon_{0}=1$. The inverse of temperature is denoted as $\beta=1 / T$. The charge and the mass of boson and fermion have unified marks as $q$ and $m$.

\section{QED PAIR PRODUCTION IN THERMAL SYSTEMS}

This method is based on motion equation, and it is convenient to take the Dirac equation and Klein-Gordon equation into the form

$$
\begin{aligned}
& \left\{\left(\partial_{\mu}-i q A_{\mu}\right)^{2}+m^{2}+2 q\left(\begin{array}{l}
(\mathbf{B}+i \mathbf{E}) \cdot \boldsymbol{\sigma}_{\mathbf{s}} \\
\quad \times \phi_{s}=0,
\end{array}\right.\right. \\
& \left.\left.\quad(\mathbf{B}-i \mathbf{E}) \cdot \boldsymbol{\sigma}_{\mathbf{s}}\right)\right\}
\end{aligned}
$$

where $\mathbf{B}$ and $\mathbf{E}$ are magnetic and electric fields and $\sigma_{\mathrm{s}}$ is half of Pauli matrix for a fermion and zero matrix for a boson. In this equation, the difference depends on their spin, where spin $s= \pm 1 / 2$ for a spinor QED and spin $s=$ 0 for a scalar QED.

\section{A. QED pair production at zero temperature}

The annihilation operator of incoming state $\phi_{\mathbf{k}, \text { in }}^{(+)}$is $a_{\mathbf{k}}\left(t_{\text {in }}=-\infty\right)$ for the particle and $b_{\mathbf{k}}\left(t_{\text {in }}=-\infty\right)$ for the antiparticle state $\phi_{\mathbf{k}, \text { in }}^{(-)}$, and similarly the annihilation operators of outgoing states $\phi_{\mathbf{k} \text {,out }}^{(+)}$and $\phi_{\mathbf{k} \text {,out }}^{(-)}$are defined by $a_{\mathbf{k}}\left(t_{\text {out }}=+\infty\right)$ and $b_{\mathbf{k}}\left(t_{\text {out }}=+\infty\right)$. The subscript $\mathbf{k}$ denotes $(\mathbf{k}, s)$, including momentum and spin, and the superscript $\mathrm{s}(+)$ and (-) distinguish the positive energy and negative energy states. The incoming states and outgoing states depend on the gauge potential $A(x)$ that determines the asymptotic solutions of Eq. (1).

The in-vacuum and out-vacuum $\mid 0$, in $\rangle$ and $\mid 0$, out $\rangle$ are defined in the particle number representation as

$$
\left.\mid 0, \text { in }\rangle=\prod_{\mathbf{k}} \otimes \mid 0_{\mathbf{k}}, \text { in }\right\rangle
$$

and

$$
\left.\left.\mid 0, \text { out }\rangle=\prod_{\mathbf{k}} U_{\mathbf{k}}\left(A^{e x}\right) \mid 0, \text { in }\right\rangle=U\left(A^{e x}\right) \mid 0, \text { in }\right\rangle .
$$

The $U\left(A^{e x}\right)$ is an evolution operator and satisfies

$$
a_{\mathbf{k}, \text { out }}=U_{\mathbf{k}}\left(A^{e x}\right) a_{\mathbf{k}, \text { in }} U_{\mathbf{k}}^{+}\left(A^{e x}\right) .
$$

The outgoing operators and ingoing operators are related through the Bogoliubov transformation

$$
\begin{aligned}
a_{\mathbf{k}, \text { out }}= & \int \frac{d^{3} \mathbf{k}^{\prime}}{2 E_{\mathbf{k}}(2 \pi)^{3}}\left[\left\langle\phi_{\mathbf{k}, \text { out }}^{(+)} \mid \phi_{\mathbf{k}^{\prime}, \text { in }}^{(+)}\right\rangle a_{\mathbf{k}^{\prime}, \text { in }}\right. \\
& \left.+\left\langle\phi_{\mathbf{k} \text {,out }}^{(+)} \mid \phi_{\mathbf{k}^{\prime}, \text { in }}^{(-)}\right\rangle b_{\mathbf{k}^{\prime}, \text { in }}^{+}\right], \\
b_{\mathbf{k}, \text { out }}^{+}= & \int \frac{d^{3} \mathbf{k}^{\prime}}{2 E_{\mathbf{k}}(2 \pi)^{3}}\left[\left\langle\phi_{\mathbf{k}, \text { out }}^{(-)} \mid \phi_{\mathbf{k}^{\prime}, \text { in }}^{(+)}\right\rangle a_{\mathbf{k}^{\prime}, \text { in }}\right. \\
& \left.+\left\langle\phi_{\mathbf{k}, \text { out }}^{(-)} \mid \phi_{\mathbf{k}^{\prime}, \text { in }}^{(-)}\right\rangle b_{\mathbf{k}^{\prime}, \text { in }}^{+}\right] .
\end{aligned}
$$

The four projections of the in basis and out basis are Bogoliubov coefficients that can be solved numerically by solving Eq. (1) in an iterative algorithm with fast Fourier transform [30,31] for arbitrary fields. When Eq. (1) has an analytical solution in a certain field, Eq. (5) has a compact form,

$$
\left(\begin{array}{l}
a_{\mathbf{k}, \text { out }} \\
b_{\mathbf{k}, \text { out }}^{+}
\end{array}\right)=\left(\begin{array}{cc}
\mu_{\mathbf{k}} & \nu_{\mathbf{k}}^{*} \\
\eta \nu_{\mathbf{k}} & \mu_{\mathbf{k}}^{*}
\end{array}\right)\left(\begin{array}{c}
a_{\mathbf{k}, \text { in }} \\
b_{\mathbf{k}, \text { in }}^{+}
\end{array}\right),
$$

where either $\eta=-1$ for bosons or $\eta=1$ for fermions, and the coefficients have the general form

$$
\mu_{\mathbf{k}}=\left\langle\phi_{\mathbf{k}, \text { out }}^{(+)} \mid \phi_{\mathbf{k}, \text { in }}^{(+)}\right\rangle, \quad \nu_{\mathbf{k}}^{*}=\left\langle\phi_{\mathbf{k}, \text { out }}^{(+)} \mid \phi_{\mathbf{k}, \text { in }}^{(-)}\right\rangle .
$$

These Bogoliubov coefficients satisfy the relation

$$
\left|\mu_{\mathbf{k}}\right|^{2}+\eta\left|\nu_{\mathbf{k}}\right|^{2}=1
$$

Combining the transformation relation (6) and number operator $a_{\text {in }}^{+} a_{\text {in }}$ leads to the number of produced pairs with k momentum [32]: 


$$
\mathcal{N}_{\mathbf{k}}=\left\langle 0, \text { out }\left|a_{\mathbf{k}, \text { in }}^{+} a_{\mathbf{k}, \text { in }}\right| 0, \text { out }\right\rangle=\left|\nu_{\mathbf{k}}\right|^{2} .
$$

Finally, the total number of produced pairs is

$$
\mathcal{N}=\int \frac{d \mathbf{k}}{(2 \pi)^{3}}\left|\nu_{\mathbf{k}}\right|^{2}
$$

\section{B. QED pair production in thermal charged particles}

In thermal QED systems, real charged particles condense when spontaneous vacuum breaking occurs, even under an external current. Here, virtual particles are ignored for we only consider one loop. Systems are assumed to be the ideal canonical ensemble, in which the chemical potential is ignored. The thermal vacuum is defined as $[33,34]$

$$
\left.\left.\mid 0, \beta_{q}, \text { in }\right\rangle=\sum_{\mathbf{k}} \sum_{n_{\mathbf{k}}} Z_{\text {in }}^{-1 / 2} e^{-\beta_{q} n_{\mathbf{k}} \varepsilon_{\mathbf{k}} / 2} \mid n_{\mathbf{k}}, \tilde{n}_{\mathbf{k}}, \text { in }\right\rangle,
$$

where $n_{\mathbf{k}}$ and $\tilde{n}_{\mathbf{k}}$ are the numbers of particles corresponding to $a_{\mathbf{k}, \text { in }}$ and $\tilde{a}_{\mathbf{k} \text {,in }}$, respectively, and satisfy $n_{\mathbf{k}}=\tilde{n}_{\mathbf{k}}$. The thermal vacuum as a thermal rotation of the in-vacuum in which $U(\theta) \mid 0$, in $\rangle=\mid 0, \beta_{q}$, in $\rangle$, where

$$
U(\theta)=e^{-\sum_{\mathbf{k}} \theta_{\mathbf{k}}\left(\beta_{q}\right)\left(\tilde{c}_{\mathbf{k}, \mathrm{in}} c_{\mathbf{k}, \text { in }}-c_{\mathbf{k}, \text { in }}^{+} \tilde{c}_{\mathbf{k}, \text { in }}^{+}\right)}
$$

The relation between thermalized incoming particles and original incoming particles is

$$
\left(\begin{array}{c}
c_{\mathbf{k}, \text { in }}(\theta) \\
\tilde{c}_{\mathbf{k}, \text { in }}^{+}(\theta)
\end{array}\right)=\left(\begin{array}{cc}
\cos (\mathrm{h}) \theta_{\mathbf{k}} & -\sin (\mathrm{h}) \theta_{\mathbf{k}} \\
\eta \sin (\mathrm{h}) \theta_{\mathbf{k}} & \cos (\mathrm{h}) \theta_{\mathbf{k}}
\end{array}\right)\left(\begin{array}{c}
c_{\mathbf{k}, \text { in }} \\
\tilde{c}_{\mathbf{k}, \text { in }}^{+}
\end{array}\right),
$$

where the fermion picks trigonometric function $\sin \theta / \cos \theta$ and the boson picks hyperbolic function $\sinh \theta / \cosh \theta$. These are represented as $\sin (\mathrm{h}) \theta_{\mathbf{k}}=\frac{e^{-\beta_{q} \varepsilon_{\mathbf{k}} / 2}}{\sqrt{1+\eta e^{-\beta_{q} \varepsilon_{\mathbf{k}}}}}, \quad \cos (\mathrm{h}) \theta_{\mathbf{k}}=\frac{1}{\sqrt{1+\eta e^{-\beta_{q} \varepsilon_{\mathbf{k}}}}}$.

Note that the thermal and nonthermal annihilation operators acting on the thermal and in vacuums, respectively, must satisfy

$$
\begin{aligned}
\left.c_{\mathbf{k}, \text { in }}(\theta) \mid 0, \beta_{q}, \text { in }\right\rangle & \left.=\tilde{c}_{\mathbf{k}, \text { in }}(\theta) \mid 0, \beta_{q}, \text { in }\right\rangle=0, \\
\left.c_{\mathbf{k}} \mid 0, \text { in }\right\rangle & \left.=\tilde{c}_{\mathbf{k}} \mid 0, \text { in }\right\rangle=0 .
\end{aligned}
$$

Since all operators of dynamical observables consist of only nontilde operators, the number operator is just $c^{+} c$. Now, we construct a doubling thermal vacuum,

$$
\begin{aligned}
\left.\mid 0,0, \beta_{q}, \text { in }\right\rangle= & \sum_{\mathbf{k}} \sum_{n_{\mathbf{k}}} \sum_{m_{\mathbf{k}}} \delta_{n_{\mathbf{k}}, m_{\mathbf{k}}} Z_{\text {in }}^{-1 / 2} e^{-\beta_{q}\left(n_{\mathbf{k}}+m_{\mathbf{k}}\right) \varepsilon_{\mathbf{k}} / 2} \\
& \left.\left.\times \mid n_{\mathbf{k}}, \tilde{n}_{\mathbf{k}}, \text { in }\right\rangle \oplus \mid m_{\mathbf{k}}, \tilde{m}_{\mathbf{k}}, \text { in }\right\rangle,
\end{aligned}
$$

where $m_{\mathbf{k}}$ is the number of antiparticles corresponding to $b_{\mathbf{k}, \text { in }}$ and $\delta_{n_{\mathbf{k}}, m_{\mathbf{k}}}$ presents charge conservation in every pure state. We denote particles with $\mathbf{a}$ and antiparticles with $\mathbf{b}$, where $\tilde{\mathbf{a}}$ and $\tilde{\mathbf{b}}$ are corresponding tilde particles. Because Eq. (15) shows that a tilde particle is produced when a particle annihilates in thermal vacuum, the relation between tilde annihilation operators $\tilde{a}$ and $\tilde{b}$ has inverse results compared with Eq. (6):

$$
\left(\begin{array}{c}
\tilde{b}_{\mathbf{k}, \text { out }} \\
\tilde{a}_{\mathbf{k}, \text { out }}^{+}
\end{array}\right)=\left(\begin{array}{cc}
\mu_{\mathbf{k}} & \nu_{\mathbf{k}}^{*} \\
\eta \nu_{\mathbf{k}} & \mu_{\mathbf{k}}^{*}
\end{array}\right)\left(\begin{array}{c}
\tilde{b}_{\mathbf{k}, \text { in }} \\
\tilde{a}_{\mathbf{k}, \text { in }}^{+}
\end{array}\right) .
$$

Then, combining Eqs. (6), (13), and (17), the transformation of incoming particles under thermal rotation and external electromagnetic fields is

$$
\left(\begin{array}{c}
a_{\mathbf{k}, \text { out }}(\theta) \\
\tilde{a}_{\mathbf{k}, \text { out }}^{+}(\theta) \\
b_{\mathbf{k}, \text { out }}^{+}(\theta) \\
\tilde{b}_{\mathbf{k}, \text { out }}(\theta)
\end{array}\right)=\left(\begin{array}{cccc}
\mu_{\mathbf{k}} \cos (\mathrm{h}) \theta_{\mathbf{k}} & -\mu_{\mathbf{k}} \sin (\mathrm{h}) \theta_{\mathbf{k}} & \nu_{\mathbf{k}}^{*} \cos (\mathrm{h}) \theta_{\mathbf{k}} & -\nu_{\mathbf{k}}^{*} \sin (\mathrm{h}) \theta_{\mathbf{k}} \\
\eta \mu_{\mathbf{k}}^{*} \sin (\mathrm{h}) \theta_{\mathbf{k}} & \mu_{\mathbf{k}}^{*} \cos (\mathrm{h}) \theta_{\mathbf{k}} & \nu_{\mathbf{k}} \sin (\mathrm{h}) \theta_{\mathbf{k}} & \eta \nu_{\mathbf{k}} \cos (\mathrm{h}) \theta_{\mathbf{k}} \\
\eta \nu_{\mathbf{k}} \cos (\mathrm{h}) \theta_{\mathbf{k}} & -\eta \nu_{\mathbf{k}} \sin (\mathrm{h}) \theta_{\mathbf{k}} & \mu_{\mathbf{k}}^{*} \cos (\mathrm{h}) \theta_{\mathbf{k}} & -\mu_{\mathbf{k}}^{*} \sin (\mathrm{h}) \theta_{\mathbf{k}} \\
\eta \nu_{\mathbf{k}}^{*} \sin (\mathrm{h}) \theta_{\mathbf{k}} & \nu_{\mathbf{k}}^{*} \cos (\mathrm{h}) \theta_{\mathbf{k}} & \eta \mu_{\mathbf{k}} \sin (\mathrm{h}) \theta_{\mathbf{k}} & \mu_{\mathbf{k}} \cos (\mathrm{h}) \theta_{\mathbf{k}}
\end{array}\right)\left(\begin{array}{c}
a_{\mathbf{k}, \text { in }} \\
\tilde{a}_{\mathbf{k}, \text { in }}^{+} \\
b_{\mathbf{k}, \text { in }}^{+} \\
\tilde{b}_{\mathbf{k}, \text { in }}
\end{array}\right)
$$

This transformation can be expressed as $T_{A B \text {,out }}(\theta)=$ $U(\theta) U\left(A^{e x}\right) T_{A B \text {,in }} U^{+}\left(A^{e x}\right) U^{+}(\theta)$. So we can calculate the number of created pairs in thermal vacuum when $t \rightarrow \infty$ as

$$
\begin{aligned}
\mathcal{N}_{\mathbf{k}, \text { out }}\left(\beta_{q}\right)= & \left\langle 0, \beta_{q}, \text { out }\left|a_{\mathbf{k}, \text { in }}^{+} a_{\mathbf{k}, \text { in }}\right| 0, \beta_{q}, \text { out }\right\rangle \\
= & \langle 0, \text { in }| U^{+}(\theta) U^{+}\left(A^{e x}\right) a_{\mathbf{k}, \text { in }}^{+} a_{\mathbf{k}, \text { in }} \\
& \left.\times U\left(A^{e x}\right) U(\theta) \mid 0, \text { in }\right\rangle \\
= & \left|\mu_{\mathbf{k}}\right|^{2} \sin (\mathrm{h})^{2} \theta_{\mathbf{k}}+\left|\nu_{\mathbf{k}}\right|^{2} \cos (\mathrm{h})^{2} \theta_{\mathbf{k}}
\end{aligned}
$$

And there are particles condensing in initial thermal vacuum when $t \rightarrow-\infty$, where

$\mathcal{N}_{\mathbf{k}, \text { in }}\left(\beta_{q}\right)=\left\langle 0, \beta_{q}\right.$, in $\left|a_{\mathbf{k}, \text { in }}^{+} a_{\mathbf{k}, \text { in }}\right| 0, \beta_{q}$, in $\rangle=\sin (\mathrm{h})^{2} \theta_{\mathbf{k}}$.

So, the number of created pairs is

$$
\Delta \mathcal{N}_{\mathbf{k}}\left(T_{q}\right)=\mathcal{N}_{\mathbf{k}, \text { out }}\left(\beta_{q}\right)-\mathcal{N}_{\mathbf{k}, \text { in }}\left(\beta_{q}\right)=\left\{\begin{array}{l}
\left|\nu_{\mathbf{k}}\right|^{2}\left(1+2 n_{\mathbf{k}}^{B}\right) \\
\left|\nu_{\mathbf{k}}\right|^{2}\left(1-2 n_{\mathbf{k}}^{F}\right)
\end{array},\right.
$$


where $n_{\mathbf{k}}^{B / F}$ denotes either the Bose-Einstein distribution $n_{\mathbf{k}}^{B}=1 /\left(e^{\beta_{q} \varepsilon_{\mathbf{k}}}-1\right)$ for scalar QED or $n_{\mathbf{k}}^{F}=1 /\left(e^{\beta_{q} \varepsilon_{\mathbf{k}}}+1\right)$ for spinor QED. This is consistent with Ref. [35]. In Eq. (21), the factor $\left|\nu_{\mathbf{k}}\right|^{2}$ response to the transformation under external fields and $2\left|\nu_{\mathbf{k}}\right|^{2} \bar{n}_{\mathbf{k}}^{B / F}$ arises due to the present thermal charged particles. The meaning of Eq. (21) conforms to the Bose-Einstein condensation for the boson and the Pauli blocking for the fermion.

When the density of particles and antiparticles is unequal or does not reach equilibrium density, the system can be in thermal but not in chemical equilibrium. We can redefine the distribution functions by a replacement $f^{ \pm}=\lambda^{ \pm} n^{ \pm}$ as Ref. [36], where superscript \pm represent particles/ antiparticles and $\lambda^{ \pm}$are fugacity factors for describing the deviation from chemical equilibrium, and add the chemical potential in density functions $n^{ \pm}=1 /\left(e^{\beta_{q}(\varepsilon \mp \mu)}+\eta\right)$. Taking these replacements into Eqs. (14) and (18), we get

$$
\begin{aligned}
\Delta \mathcal{N}_{\mathbf{k}}\left(T_{q}, \kappa\right) & =\left|\nu_{\mathbf{k}}\right|^{2}\left(1-\eta\left(\lambda^{-} n_{\mathbf{k}}^{-}+\lambda^{+} n_{\mathbf{k}}^{+}\right)\right) \\
& =\left\{\begin{array}{l}
\left|\nu_{\mathbf{k}}\right|^{2}\left(1+\left(\lambda^{-} n_{\mathbf{k}}^{B,-}+\lambda^{+} n_{\mathbf{k}}^{B,+}\right)\right) \\
\left|\nu_{\mathbf{k}}\right|^{2}\left(1-\left(\lambda^{-} n_{\mathbf{k}}^{F,-}+\lambda^{+} n_{\mathbf{k}}^{F,+}\right)\right)
\end{array} .\right.
\end{aligned}
$$

When $\lambda^{+}=\lambda^{-}=1$, total charges are conservative, and the presence of the chemical potential enhances the thermal correction for $n_{\mathbf{k}}^{-}+n_{\mathbf{k}}^{+}>2 n_{\mathbf{k}}$. The fugacity factors depend on the ratio of the experimental to equilibrium density [37].

Note that what we calculate is the number of incoming particles $a_{\text {in }}$, which is not thermalized particles $a_{\text {in }}(\theta)$. Because the thermal transformation is equivalent to the Kubo-Martin-Schwinger condition, the thermal particles $a(\theta)$ satisfy the corresponding condition, i.e., $\phi(t)=$ $\pm \phi(t-i \beta)$. However, it is a basic condition in early imaginary-time effective Lagrangian approaches at finite temperature, in which the asymptotic particles are also assumed to meet this condition. So, the number of thermalized particles is

$$
\left\langle 0, \beta_{q}, \text { out }\left|a_{\mathbf{k}, \text { in }}^{+}(\theta) a_{\mathbf{k}, \text { in }}(\theta)\right| 0, \beta_{q}, \text { out }\right\rangle=\left|\nu_{\mathbf{k}}\right|^{2} .
$$

This is just the result at zero temperature, so it may indirectly explain why some early discussions infer the absence of thermal corrections in pair production in one loop at finite temperature.

\section{Tree-level correction of thermal photons}

Once charged pairs appear, thermal photons emerge as heat mediums in equilibrium QED systems. Inspired by Ref. [38], which discusses the change of mass when pairs created in the periodic oscillating electromagnetic fields, it is found that the thermal Abelian gauge fields also change the mass of charged particles, which results in a vacuum inequivalent to the original one. In this consideration, we assume that the total background fields consist of external fields and thermal fields, or, more generally, any electromagnetic fields can be divided into

$$
A_{\mu}\left(x, T_{\gamma}\right)=A_{\mu}^{e x}(x)+A_{\mu}^{\gamma}\left(T_{\gamma}\right),
$$

with the assumption

$$
\left[A_{\mu}^{e x}(x), A_{\mu}^{\gamma}(T)\right]=0,
$$

which means that the two fields are both classical fields and only tree-level Feynman diagrams are included. We assume that the external field is strong and the thermal field is weak, so the two kinds of fields are absolutely separated, and nonlinear terms are ignored.

Substituting Eq. (24) in Eq. (1) and averaging it, all linear terms will be offset, for thermal photons are isotropic. Only $-q^{2}\left\langle A^{\gamma, \mu} A_{\mu}^{\gamma}\right\rangle$ is left and is equivalent to a mass shift in Eq. (1):

$$
m^{* 2}=m^{2}-q^{2}\left\langle A^{\gamma, \mu} A_{\mu}^{\gamma}\right\rangle .
$$

This $A^{\gamma}(T)$ is assumed to be the sum of linear free photon fields, so the square term is proportional to invariant density $n_{\omega} / 2 \omega$. After integrating, see the Appendix in detail, we obtain

$$
m^{* 2}=m^{2}+\frac{q^{2} T^{2}}{12} .
$$

The square root of this shift part was also obtained in Ref. [39] and called the effective mass of thermal photons [40]. Before further discussions, we denote that $m_{+}^{*}=$ $m^{*}=m+m_{\beta}$ and $m_{-}^{* 2}=m^{2}-q^{2} T^{2} / 12$ and $m_{-}^{*} \approx m-m_{\beta}$. Next, we will indicate that different asymptotic time conditions of thermal fields would lead to opposite thermal corrections.

Now, we constrain a condition $A^{\gamma}(t= \pm \infty) \neq 0$, meaning thermal fields are always existing and can be treated as a static heat bath. Hence, $\mid 0$, in; $\left.m_{+}^{*}\right\rangle$ and $\mid 0$, out; $\left.m_{+}^{*}\right\rangle$ are the dressed states in vacuum and out vacuum, where $m_{+}^{*}$ denotes that physical mass $m$ of charged particles is directly substituted by dressed mass $m_{+}^{*}$. The corresponding number operator is $\mathcal{N}_{\mathbf{k}}\left(T_{\gamma}\right)=a_{\mathbf{k}, \text { in } ; m_{+}^{*}} a_{\mathbf{k}, \text { in } ; m_{+}^{*}}$, so what we calculate is the number of dressed particles

$N_{\mathbf{k}}\left(T_{\gamma}\right)=\left\langle 0\right.$, out; $m_{+}^{*}\left|\mathcal{N}_{\mathbf{k}}\left(T_{\gamma}\right)\right| 0$, out $\left.; m_{+}^{*}\right\rangle=\left|\nu_{\mathbf{k}}\right|_{m \rightarrow m_{+}^{*}}^{2}$.

This means the threshold has increased $\left(E_{+}^{*}=2 m_{+}^{*} c^{2}>\right.$ $2 m c^{2}$ ) and more energy is demanded to counteract the oscillating force turning into potential energy of dressed particles.

If we adopt a weaker condition $A^{\gamma}(t=+\infty)=0$, the number operator should be a unaltered quantity which 
corresponds to the final measurement. This is to make the physical mass $m$ unaltered so that the measured mass is equal to physical mass. In a physical process, we can think that the pairs absorb surrounding thermal photons; consequently, the $A^{\gamma}$ vanish when $t \rightarrow \infty$. In other words, the out vacuum is supposed to in an equivalent measured Fock space [34], or has the same mass term in the Lagrangian compared with original one, and is denoted by $\mid 0$, out $\rangle$, corresponding the vacuum after evolution under $A^{e x}$ and $A^{\gamma}$. For the spinor, the physical mass is determined by $\left.(\not \supset-m)\left(\not \not-m_{-}^{*}-m_{\beta}\right)^{-1}\right|_{\not \supset=m}=1$, and the equivalent mass is obtained: $m_{-}^{*}=m-m_{\beta}$. Then, the equivalent Lagrangian is $\mathcal{L}=i \bar{\psi} \not \supset \psi-m \bar{\psi} \psi+m_{\beta} \bar{\psi} \psi$, where the third term is treated as a kinetic term. The out vacuum is

$$
\left.\mid 0, \text { out }\rangle \approx \mathcal{T} e^{i \int d^{4} x \bar{\psi}(i \not D-m) \psi} e^{i \int^{\Omega} d^{4} x m_{\beta} \bar{\psi} \psi} \mid 0, \text { in }\right\rangle,
$$

where $\int^{\Omega} d^{4} x=\int d^{3} x \int^{\Omega} d t$ and $\Omega$ is the turn-off time of thermal fields. In addition, the in vacuum can be represented as the evolution of another in vacuum:

$$
\left.\mid 0, \text { in }\rangle=\mathcal{T} e^{-i \int^{\Omega} d^{4} x m_{\beta} \bar{\psi} \psi} \mid 0, \text { in } ; m_{-}^{*}\right\rangle .
$$

Upon inserting it into (29) with $m=m_{-}^{*}+m_{\beta}$, we obtain

$$
\begin{aligned}
\mid 0, \text { out }\rangle \approx & \mathcal{T}\left(e^{i \int d^{4} x \bar{\psi}\left(i \not D-m_{-}^{*}\right) \psi} e^{i \int_{\Omega} d^{4} x \bar{\psi} \overline{\bar{i} \bar{j}_{\beta}(x)} \psi}\right) \\
& \left.\times\left.\left(e^{i \int d^{\Omega} x \bar{\psi}_{\bar{i} \bar{\delta} \delta_{\beta}(x)}(x)} e^{-i \int d^{4} x m_{\beta} j_{\beta}(x)}\right)\right|_{j_{\beta}(x)=0} \mid 0, \text { in } ; m_{-}^{*}\right\rangle \\
= & \left.U\left(A^{e x}\right) \times U\left(A^{\gamma} \mid A^{e x}\right) \mid 0, \text { in } ; m_{-}^{*}\right\rangle,
\end{aligned}
$$

where the $j_{\beta}(x)$ is the external source of thermal fields and is independent of the in vacuum as $\left(\delta / \delta j_{\beta}\right) \mid 0$, in $\rangle=0$. In the above calculations, we use Eq. (27) and the assumption that $A^{\gamma}\left(T_{\gamma}\right)$ is small, so relation $e^{A+B} \approx e^{A} e^{B}$ is used. In last line of (31), the total evolution operator is divided into two parts: the $U\left(A^{e x}\right)$, which undertakes the main evolution of the vacuum, and $U\left(A^{\gamma} \mid A^{e x}\right)$, which changes the asymptotic structure of the vacuum. It is obvious that

$$
\begin{aligned}
\left.U\left(A^{e x}\right) \mid 0, \text { in } ; m_{-}^{*}\right\rangle & \left.=\mid 0, \text { out } ; m_{-}^{*}\right\rangle, \\
\left.U\left(A^{\gamma} \mid A^{e x}\right) \mid 0, \text { out } ; m_{-}^{*}\right\rangle & =\mid 0, \text { out }\rangle .
\end{aligned}
$$

And the commutative relation $\left[U\left(A^{\gamma} \mid A^{e x}\right), U\left(A^{e x}\right)\right]=0$ holds due to Eq. (25); therefore, the evolutionary order does not affect the final result. Furthermore, $U\left(A^{\gamma} \mid A^{e x}\right)$ is a unitary operator, for its power is purely imaginary. Applying the above analysis and Eq. (30), we get that

$$
\begin{aligned}
& \left.U^{+}\left(A^{\gamma} \mid A^{e x}\right) a_{\mathbf{k}, \text { in }} U\left(A^{\gamma} \mid A^{e x}\right) U^{+}\left(A^{\gamma} \mid A^{e x}\right) \mid 0, \text { in }\right\rangle \\
& \left.\quad=a_{\mathbf{k}, \text { in } ; m_{-}^{*}} \mid 0, \text { in; } m_{-}^{*}\right\rangle=0 .
\end{aligned}
$$

Now, with Eqs. (31) and (33), we can obtain the number of produced pairs in this scenario as

$$
\begin{aligned}
N_{\mathbf{k}}\left(T_{\gamma}\right)= & \left\langle 0, \text { out }\left|a_{\mathbf{k}, \text { in }}^{+} a_{\mathbf{k}, \text { in }}\right| 0, \text { out }\right\rangle \\
= & \left\langle 0, \text { in } ; m_{-}^{*}\right| U^{+}\left(A^{e x}\right) U^{+}\left(A^{\gamma} \mid A^{e x}\right) a_{\mathbf{k}, \text { in }}^{+} a_{\mathbf{k}, \text { in }} \\
& \left.\times U\left(A^{\gamma} \mid A^{e x}\right) U\left(A^{e x}\right) \mid 0, \text { in } ; m_{-}^{*}\right\rangle \\
= & \left\langle 0, \text { out } ; m_{-}^{*}\left|a_{\mathbf{k}, \text { in } ; m_{-}^{*}}^{+} a_{\mathbf{k}, \text { in } ; m_{-}^{*}}\right| 0, \text { out } ; m_{-}^{*}\right\rangle \\
= & \left|\nu_{\mathbf{k}}\right|_{m \rightarrow m_{-}^{*}}^{2} .
\end{aligned}
$$

This result also works for a scalar with similar procedures. Note that when $U\left(A^{e x}\right)$ acts on the vacuum the result depends on the asymptotic structure of the vacuum, and so does $U(\theta)$. This means that in order to take the tree-level correction of thermal photons into pair production, we only need to do a mass shift in the Bogoliubov coefficient, so the threshold has gone down $\left(E_{-}^{*}=2 m_{-}^{*} c^{2}<2 m c^{2}\right)$, which verifies our guess that the dressing of thermal photons enhances pair production. In subsequent discussions, we will adopt this approach.

More generally, stable equilibrium QED systems should contain both thermal charged particles and thermal photons. Following the above approaches, the thermal out vacuum also is an equivalent measured thermal vacuum,

$$
\left.\left.\mid 0,0, \beta_{q}, \text { out }\right\rangle=U\left(A^{\gamma} \mid A^{e x}\right) U\left(A^{e x}\right) U(\theta) \mid 0,0, \text { in; } m_{-}^{*}\right\rangle .
$$

Then, we do similar calculations as in Eq. (34), and pair production in this situation is obtained as

$$
N_{\mathbf{k}}\left(T_{q}, T_{\gamma}\right)=\left[\left|\nu_{\mathbf{k}}\right|^{2}\left(1 \pm n_{\mathbf{k}}^{B / F}\right)\right]_{m \rightarrow m_{-}^{*}} .
$$

This result is just Eq. (21) with a negative mass shift, which means that the tunneling particles and thermal particles all have absorbed thermal photons. A remark is that the $\bar{n}_{\mathbf{k}}^{F / B}$ in Eq. (36) depends on not only charged particles temperature $T_{q}$ in the Boltzmann factor $e^{\varepsilon_{\mathbf{k}} / T_{q}}$ but also on thermal photons temperature $T_{\gamma}$ in $\varepsilon_{\mathbf{k}}=\sqrt{m^{2}-q^{2} T_{\gamma}^{2} / 12+\mathbf{k}^{2}}$. Here, we ignored any nonlinear and high terms due to the assumptions that $T_{\gamma}$ is small $q^{2} T_{\gamma}^{2} / 12 \ll m^{2}$ and that $m_{-}^{* 2}$ is always positive.

Note that now we have obtained the general formal results of Schwinger pair production for the arbitrary external fields at finite temperature. Our study has taken into account the tree-level correction of real thermal photons, which is a useful complementary to the previous works [5-9,12,21-23].

\section{CONSTANT ELECTRIC FIELD}

In this section, we apply the formal results above to an external constant electric field. Before considering finite temperature, we review the solutions of Eq. (1) in a 
constant electric field [24]. In the time-dependent gauge $A_{z}=-E t$, Eq. (1) takes the form

$$
\left[\partial_{t}^{2}+m^{2}+\mathbf{k}_{\perp}^{2}+\left(k_{z}-q E t\right)^{2}+2 i s q E\right] \phi_{\omega, \mathbf{k}}(t)=0 .
$$

When $t=-\infty$, the solution is expressed in terms of the parabolic cylinder functions

$$
\phi_{\omega, \mathbf{k}}(t)=D_{p}(z) .
$$

And when $t=\infty$, it turns to

$D_{p}(z)=e^{-i p \pi} D_{p}(-z)+\frac{\sqrt{2 \pi}}{\Gamma(-P)} e^{-i(p+1) \pi / 2} D_{-p-1}(i z)$,

where

$$
\begin{aligned}
& z=\sqrt{\frac{2}{q E}} e^{i \pi / 4}\left(k_{z}-q E t\right), \\
& p=-\frac{1}{2}-i \frac{m^{2}+\mathbf{k}_{\perp}^{2}+2 i s q E}{2 q E} .
\end{aligned}
$$

The Bogoliubov coefficients are

$$
\mu_{\mathbf{k}}=\frac{\sqrt{2 \pi}}{\Gamma(-p)} e^{-i(p+1) \pi / 2}, \quad \nu_{\mathbf{k}}=e^{i p \pi}
$$

So, the total number of produced pairs is

$$
\begin{aligned}
N\left(E, T_{q}=T_{r}=0\right) & =\sum_{\mathbf{k}}\left|\nu_{\mathbf{k}}\right|^{2} \\
& =\frac{q E}{4 \pi(1-|s|)} \int \frac{d^{2} k_{\perp}}{(2 \pi)^{2}} e^{-\frac{\pi\left(m^{2}+k_{\perp}^{2}\right)}{q E}} \\
& =\frac{q^{2} E^{2}}{16(1-|s|) \pi^{3}} e^{-\frac{\pi m^{2}}{q E}},
\end{aligned}
$$

where $\frac{q E}{4 \pi(1-|s|))}$ is the number of states along the $z$ direction and Eq. (42) is consistent with Schwinger's result [1] when $|s|=1 / 2$ for the spinor.

In the following, thermal gauge fields are assumed to be turned off when $t \rightarrow \infty$ as long as they are considered. Meanwhile, we assume that all systems are in equilibrium initially, although an external constant field is not good to get asymptotically free states. In the limits of low temperature and low density, the thermal dissipation and backreaction of the created pairs are both neglected. In other words, systems are thermalized initially, but the process of producing pairs is dynamic.

$$
\text { A. } T_{q}=\mathbf{0}, T_{\gamma} \neq 0
$$

Now, we consider a pure thermal photon system with a constant electric field, where the low-temperature limit $q^{2} T_{\gamma}^{2} / 12 \ll m^{2}$ is reasonable since no real particles exist initially due to low quantum fluctuation. But the tunneling particles are also dressed by thermal photons. In this way, the number of produced pairs is obtained by substituting $\mathrm{m}^{2}$ in Eq. (42) with $m^{2}-q^{2} T_{\gamma}^{2} / 12$ according to Eq. (34),

$$
N\left(E, T_{\gamma}\right)=\frac{(q E)^{2}}{16(1-|s|) \pi^{3}} e^{\frac{-\pi m^{2}}{q E}} e^{\frac{\pi q T_{\gamma}^{2}}{12 E}}
$$

At small $T_{\gamma}$, this result has a series expanded form,

$$
\begin{aligned}
N\left(E, T_{\gamma}\right)= & N\left(E, T_{q}=T_{\gamma}=0\right) \\
& \times\left(1+\frac{\pi q T_{\gamma}^{2}}{12 E}+\frac{1}{2}\left(\frac{\pi q}{12 E}\right)^{2} T_{r}^{4}+\cdots\right) .
\end{aligned}
$$

The exponent $T_{\gamma}^{2} / E$ means that the stronger electric field is, the less effect fixed thermal photons causes. In Ref. [41], thermal photons are considered by two-loop calculation, and the lowest-order thermal correction term contains $T^{4}$, but we get $T^{2}$. This is because the thermal fields are treated as an external current in our discussion, while those are internal in Ref. [41], in which more double vertices in diagram expansion exist.

The numerical result of Eq. (43) is shown in Fig. 1. Note that the theoretical formula is valid under the lowtemperature limit $T_{\gamma} \ll \sqrt{3 / \pi \alpha} m c^{2} / k_{B} \approx 11 T_{c}$, where $T_{c}=m c^{2} / k_{B}$. Figure. 1 shows that the enhancement of pair production is small, even if the photons temperature is 8 times of critical temperature, see the top yellow line.

$$
\text { B. } T_{q} \neq 0, T_{\gamma}=0
$$

In this physical situation, the temperature $T_{q}$ of thermal charged particles is not supposed to be high enough to produce real photons. Then, with a constant electric field, the result is obtained directly by combining Eqs. (21) and (42), that is,

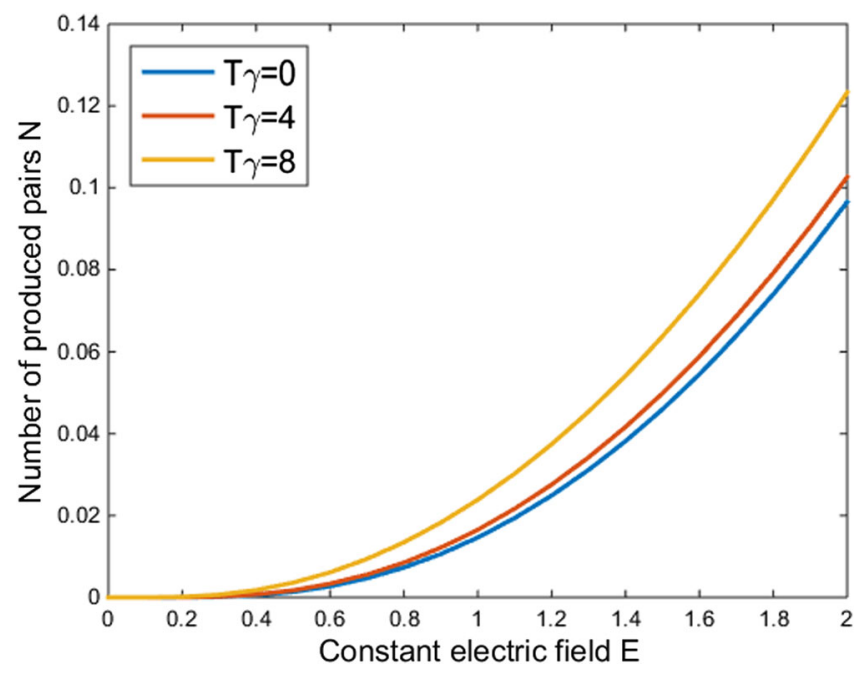

FIG. 1. Number of produced pairs for $1 / 2$ spinor fermion depends on the constant electric field at three different photon temperatures. Here the dimensionless parameters $k_{B} T_{\gamma} / m c^{2} \rightarrow$ $T_{\gamma}$ and $q E \hbar / \pi m^{2} c^{3} \rightarrow E$ are used. 

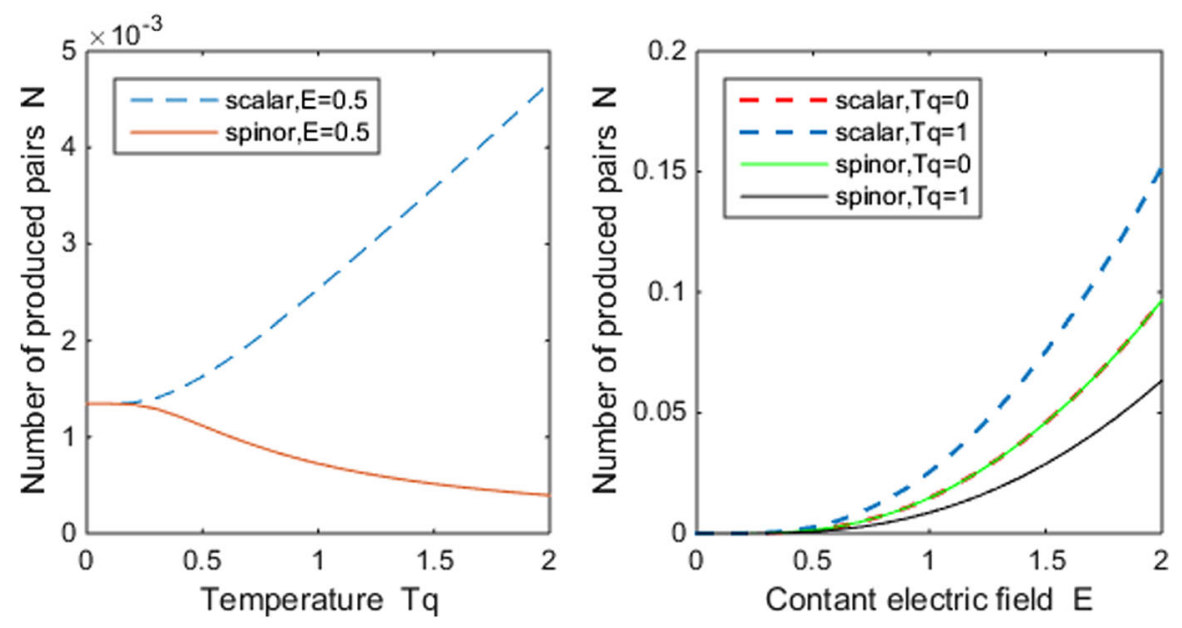

FIG. 2. The number of scalar (dashed line) and spinor (solid line) pairs is plotted as a function of $T_{q}$ and $E$, respectively [see Eq. (45)]. The normalized scheme is the same as in Fig. 1. Note that for a good eye guiding, the number of scalar pairs is doubled for keeping the same starting when $T_{q}=0$.

$$
\begin{aligned}
N\left(E, T_{q}\right)= & \frac{q E}{4 \pi(1-|s|)} \int \frac{d^{2} k_{\perp}}{(2 \pi)^{2}} e^{-\frac{\pi\left(m^{2}+k_{\perp}^{2}\right)}{q E}} \\
& \times\left(1-\frac{2 \eta}{e^{\sqrt{m^{2}+k_{\perp}^{2}} / T_{q}}+\eta}\right) .
\end{aligned}
$$

Here, the longitudinal momentum of initial particles is assumed to be zero, where an extra force is constructed to neutralize the electric field force. As plotted in Fig. 2, it returns to (42) when $T_{q} \rightarrow 0$ but tends to either infinity for bosons or zero for fermions when $T_{q} \rightarrow \infty$. On the other hand, from Fig. 2, one can see that the monotonous dependence of created scalar or spinor pairs number to the external field is a little larger or smaller for thermal case than that of nonthermal case. When we return to $T_{q} \ll m$, the number of produced pairs (45) has an approximate form:

$$
\begin{aligned}
N\left(E, T_{q}\right)= & \frac{(q E)^{2}}{16(1-|s|) \pi^{3}} e^{-\pi m^{2} /(q E)} \\
& \times\left(1-\eta \frac{q E}{4 \pi^{2}} e^{-\pi m^{2} /(q E)-m / T_{q}}\right) .
\end{aligned}
$$

$$
\text { C. } T_{q}=T_{\gamma} \neq \mathbf{0}
$$

Finally, we assume that the initial systems are in absolute equilibrium in which thermal photons and charged particles have the same temperature $T_{q}=T_{\gamma}=T$ with an external constant electric field; the system is actually an ideal particle-antiparticle-photon gas. Combining Eqs. (45) and (36), the number of produced pairs is obtained as

$$
\begin{aligned}
N\left(E, T_{q}=T_{\gamma}=T\right)= & \frac{q E}{4 \pi(1-|s|)} \int \frac{d^{2} k_{\perp}}{(2 \pi)^{2}} e^{-\frac{\pi\left(m^{2}+k_{\perp}^{2}\right)}{q E}+\frac{\pi T^{2}}{12 E}} \\
& \times\left(1-\frac{2 \eta}{e^{\beta \sqrt{m^{2}-q^{2} T^{2} / 12+k_{\perp}^{2}}}+\eta}\right),
\end{aligned}
$$

which is plotted in Fig. 3, meanwhile, by the comparison with Eq. (43) and Eq. (45), the other numerical results are included. The figure shows that the correction of thermal photons is much less than the correction by thermal pairs. Of course Eq. (47) has an approximate form at small $T$,

$$
\begin{aligned}
N\left(E, T_{q}=T_{\gamma}=T \ll m\right)= & \frac{q E}{4(1-|s|) \pi} e^{\frac{-\pi m^{2}}{q E}} e^{\frac{\pi q T^{2}}{12 E}} \\
& \times\left(1-\eta \frac{q E}{4 \pi^{2}} e^{-\sqrt{m^{2} / T^{2}-q^{2} / 12}}\right) .
\end{aligned}
$$

So far, we have not used any limit on electric field $E$, but the low-temperature limit for $T_{\gamma}$ is necessary for our linear assumption in Sec. II. C. In fact, we expect the external field is much stronger than temperature so that the thermal dissipation and backreactions can be neglected.

\section{DISCUSSION}

In this paper, to calculate Schwinger pair production at finite temperature, we generalize the evolution operator method of TFD to generic thermal QED systems that contain not only thermal charged particles but also photons. Then, we get the thermal transformation relation of outgoing operators and incoming operators under external fields. What we have done on the basis of Eq. (1) is similar to Sauter's calculation on the Dirac equation [42], except for the part where we add thermal distribution. Then, we use an effective substitute, $m_{+}^{* 2}=m^{2}+q^{2} T^{2} / 12$, to represent the correction of thermal photons, but that has an inverse substitution when the turning off of thermal fields is considered. Lastly, applying the thermal average approach $\left\langle\right.$ out - vacuum $\left|a_{\text {in }}^{+} a_{\text {in }}\right|$ out - vacuum $\rangle$, we get QED pair 

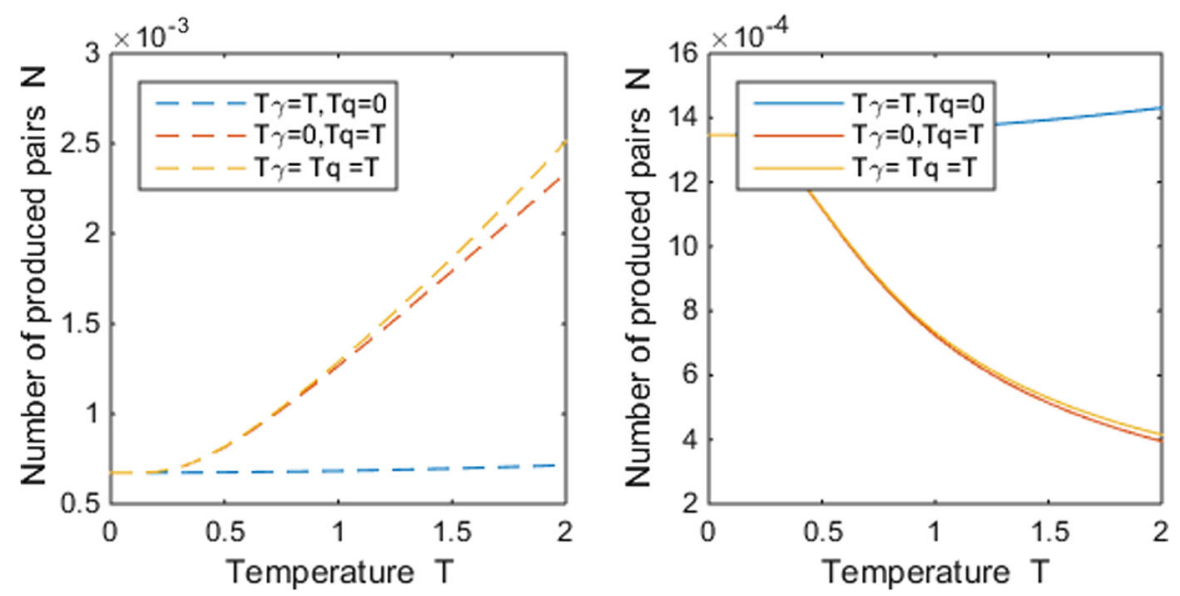

FIG. 3. The number of scalar (dashed line) and spinor (solid line) pairs are plotted as three functions [Eqs. (43), (45), and (47)] of only photons temperature $T_{\gamma}$, only pairs temperature $T_{q}$ and both temperature $T_{\gamma}=T_{q}=T$ when $E=0.5$. The normalized temperature and field are the same as in Fig. 1.

production in thermal systems. In our results, $\left|\nu_{\mathbf{k}}\right|^{2}$ corresponds to the decaying part of incoming states under the external field, and density $n_{\mathbf{k}}^{B / F}$ arises for initial thermal charged particles, and $m_{+}^{* 2}$ represents effective mass of both thermal particles and tunneling particles dressed by thermal photons. In an external constant electric field, the precise integral results and the approximate polynomial results are obtained, and they both recover Schwinger's result at $T=0$.

Back to our results, we have not used any particular fields but only the Bogoliubov coefficient $\nu_{\mathbf{k}}$ in our formal results, so it can be applied to arbitrary fields as long as we obtain the coefficient. There are no poles or infinity to be renormalized, since the number of produced pairs is an observable quantity. If charged particles or photons are not in ideal equilibrium initially, it can be completed by using a corresponding distribution function to replace the ideal one as we have done in Sec. II. B. It also can be generalized to the thermal Bethe-Heitler process $\left(\gamma Z \rightarrow e^{+} e^{-} Z\right)$ and thermal trident mechanism $\left(e^{-} Z \rightarrow\right.$ $\left.e^{-} e^{+} e^{-} Z\right)$ in which thermal massive charged particle $Z$ exists [43] and the classical Coulomb field can be taken into the background field as an equivalent approach, but the regions need to be distinguished for the Debye shielding. So, it may be helpful in relativistic plasma and heavy ion collision.

On the experimental side, it is proposed to observe thermal Schwinger pair production by a thermal bath of photons in a constant electric field [28], and our results in a constant $E$ supplement neglected correction. What's more is that our formal results can be applied to general laser fields by combining numerical simulation [44]. In addition, momentum spectrum distribution of production particles is given in our results, and spatial spectral distribution also can be obtained by Fourier transform. These results are available by comparisons with experimental data in possible future experiments.
Again, our results mainly work in low-temperature limit $T_{\gamma} \ll m$, where backreactions and dissipations have not been considered. With temperature increasing, the full equilibrium description is more appropriate, and for more detailed summary and review, one can refer to Ref. [12]. When the temperature is extremely high, the effective Lagrangian approaches are more proper, which is beyond the present study and is still an open topic problem for future research.

\section{ACKNOWLEDGMENTS}

We thank Doctor Z.L. Li for helpful discussions and M. Ababekri for his reading of the paper and modification to the language. This work was supported by the National Natural Science Foundation of China under Grants No. 11875007 and No. 11935008.

\section{APPENDIX: THERMAL MASS CORRECTION OF CHARGED PARTICLE}

In Sec. II. B, we illustrate the use of mass shift with thermal photons, that is,

$$
m^{* 2}=m^{2}+\frac{q^{2} T^{2}}{12}
$$

where $q^{2} T^{2} / 12$ is same as Eq. (35) in Ref. [45] calculated in imaginary form and Eq. (14) in Ref. [46] in real form, but different from the effective photon mass, $m_{\mathrm{eff}}=q T / 3$, calculated in one-loop polarization [47]. Now, we derive it with semiclassical approaches, beginning with

$$
m^{* 2}=m^{2}-q^{2}\left\langle A^{\gamma, \mu} A_{\mu}^{\gamma}\right\rangle,
$$

where $A_{r}$ is the linear sum of all thermal photons and \langle\rangle indicates averaging over time and space. We assume that all 
thermal photons are free and satisfy $\left\langle A^{f, \mu} A_{\mu}^{f}\right\rangle=-1 / 2 \omega$ and integrate this relation by

$$
\left\langle A^{\gamma, \mu} A_{\mu}^{\gamma}\right\rangle=-\int_{0}^{\infty} d \omega \frac{n(\omega)}{2 \omega},
$$

where $n(\omega) / 2 \omega$ is the invariant density and $n(\omega)$ is equal to

$$
n(\omega)=\frac{g(\omega)}{e^{\beta \omega}-1}=\frac{\omega^{2}}{\pi^{2}\left(e^{\beta \omega}-1\right)},
$$

where $g(\omega)$ is the energy density and its value is inserted directly here. Combining Eqs. (A2)-(A4), we obtain Eq. (A1).
[1] J. S. Schwinger, Phys. Rev. 82, 664 (1951).

[2] S. Gavrilov, D. Gitman, and A. Shishmarev, Phys. Rev. D 99, 116014 (2019).

[3] G. Dunne, Eur. Phys. J. Spec. Top. 223, 1055 (2014).

[4] A. Di. Piazza, C. Muller, K. Z. Hatsagortsyan, and C. H. Keitel, Rev. Mod. Phys. 84, 1177 (2012).

[5] O. Gould and A. Rajantie, Phys. Rev. D 96, 076002 (2017).

[6] A. Monin and M. B. Voloshin, Phys. Rev. D 81, 025001 (2010).

[7] A. R. Brown, Phys. Rev. D 98, 036008 (2018).

[8] P. Draper, Phys. Rev. D 98, 125014 (2018).

[9] L. Medina and M. C. Ogilvie, Phys. Rev. D 95, 056006 (2017).

[10] M. Korwar and A. M. Thalapillil, Phys. Rev. D 98, 076016 (2018).

[11] G. Torgrimsson, Phys. Rev. D 99, 096007 (2019).

[12] O. Gould, S. Mangles, A. Rajantie, S. Rose, and C. Xie, Phys. Rev. A 99, 052120 (2019).

[13] D. A. Kirzhnits and A. D. Linde, Phys. Lett. B 42, 471 (1972).

[14] S. Weiberg, Phys. Rev. D 9, 3357 (1974).

[15] L. Dolan and R. Jackiw, Phys. Rev. D 9, 3320 (1974).

[16] C. W. Bernard, Phys. Rev. D 9, 3312 (1974).

[17] Y. Ueda, Phys. Rev. D 23, 1383 (1981).

[18] JI. Kapusta and C. Gale, Finite-Temperature Field Theory: Principles and Applications (Cambridge University Press, Cambridge. England, 2006).

[19] H. Umezawa, Advanced Field Theory: Micro, Macro, and Thermal Physics (AIP, New York, 1995).

[20] F. Gelis and N. Tanji, Prog. Part. Nucl. Phys. 87, 1 (2016).

[21] W. Dittrich, Phys. Rev. D 19, 2385 (1979).

[22] H. Gies, Phys. Rev. D 60, 105002 (1999).

[23] P. Elmfors and B. S. Skagerstam, Phys. Lett. B 348, 141 (1995).

[24] S. P. Kim and H. K. Lee, Phys. Rev. D 76, 125002 (2007); 78, 105013 (2008).
[25] S. P. Gavrilov and D. M. Gitman, Phys. Rev. D 78, 045017 (2008).

[26] S. P. Gavrilov, D. M. Gitman, and J. L. Tomazelli, Nucl. Phys. B795, 645 (2008).

[27] P. Krekora, Q. Su, and R. Grobe, Phys. Rev. A 73, 022114 (2006).

[28] S. J. Rose, High Energy Density Phys. 9, 480 (2013).

[29] H. Gies and F. Karbstein, J. High Energy Phys. 03 (2017) 108.

[30] J. W. Braun, Q. Su, and R. Grobe, Phys. Rev. A 59, 604 (1999).

[31] A. Blinne, H. Gies, F. Karbstein, C. Kohlfurst, and M. Zepf, Phys. Rev. D 99, 016006 (2019).

[32] It is $\left\langle 0\right.$, in $\left|a_{\mathbf{k} \text {,out }}^{+} a_{\mathbf{k} \text {,out }}\right| 0$, in $\rangle$ in some literature but has no difference in the result, since the transformation is unitary.

[33] I. Ojima, Ann. Phys. (N.Y.) 137, 1 (1981).

[34] A. Das, Finite Temperature Field Theory (World Scientific, Singapore, 1997).

[35] S. P. Kim and H. K. Lee, Phys. Rev. D 79, 045024 (2009).

[36] T. S. Biro, E. van Doorn, B. Muller, M. H. Thoma, and X. N. Wang, Phys. Rev. C 48, 1275 (1993).

[37] M. H. Thoma, Eur. Phys. J. D 55, 271 (2009).

[38] C. Kohlfurst, H. Gies, and R. Alkofer, Phys. Rev. Lett. 112, 050402 (2014).

[39] M. Loewe and J. C. Rojas, Phys. Rev. D 46, 2689 (1992).

[40] T. W. B. Kibble, Phys. Rev. 150, 1060 (1966).

[41] H. Gies, Phys. Rev. D 61, 085021 (2000).

[42] F. Sauter, Z. Phys. 69, 742 (1931).

[43] I. A. Maltsev and V. M. Shabaev, Phys. Rev. Lett. 123, 113401 (2019).

[44] P. Krekora, K. Cooley, Q. Su, and R. Grobe, Phys. Rev. Lett. 95, 070403 (2005).

[45] A. Romeo, J. Math. Phys. (N.Y.) 34, 2206 (1993).

[46] G. Peressutti and B. S. Skagerstam, Phys. Lett. 110B, 406 (1982).

[47] H. A. Weldon, Phys. Rev. D 26, 1394 (1982). 